\title{
Orientación del marketing estratégico en universidades públicas
}

\section{Orientation on strategic marketing of public universities Orientaçáo do marketing estratégico nas universidades públicas} Akever Karina Santafé Rojas*

\section{RESUMEN}

El propósito del presente artículo es evidenciar la orientación del Marketing Estratégico en Universidades Públicas, sustentándose teóricamente en los planteamientos de: Sainz (2007), Munuera y Rodríguez (2007), entre otros, instaurado dentro del paradigma epistemológico post-positivista cualitativo, bajo el método fenomenológico y utilizando un muestreo por conveniencia. Luego de analizar y discutir los resultados se concluye que las Universidades Públicas orientan parte de sus acciones a la vivencia del marketing estratégico con el fin de crear posicionamiento en el mercado educativo.

Palabras clave: marketing estratégico, universidad pública, mercado, posicionamiento.

\section{ABSTRACT}

The purpose of this article is to show the orientation of Strategic Marketing in Public Universities relying on theoretical approaches: Sainz (2007), Munuera and Rodriguez (2007), among others, established withinthe post-positivist qualitative epistemological paradigmunder the phenomenological method, by convenience sampling. After analyzing and discussing the results it is concluded that public universities orient some of their shares to the experience of strategic marketing in order to create the educational market positioning.

Keywords: strategic marketing, public university, market, positioning.

\section{RESUMO}

O objetivo do presente artigo é mostrar a orientaçáo de Marketing Estratégico nas Universidades Públicas, se sustentando teoricamente nas ideias de Sainz (2007), Munuera e Rodriguez (2007), entre outros, instaurado dentro do paradigma epistemológico qualitativo pós-positivista, sob o método fenomenológico e usando uma amostra por conveniência. Após a análise e discussão dos resultados, conclui-se que as Universidades Públicas orientam parte de suas açóes à experiência do marketing estratégico com a finalidade de criar posicionamento no mercado da educação.

Palavras-chave: Marketing Estratégico, Universidade Pública, mercado, posicionamento.

Colombiana. Posdoctorado en Gerencia Pública y Gobierno (en curso), Doctorado en Ciencias Gerenciales, Maestría en Gerencia de Empresas Mención Mercadeo, Administrador de Empresas, especialista en Gestión Educativa, Licenciada en Comercio, Universidad de Pamplona, Colombia. Correspondencia con la autora: karisan19@hotmail.com. 


\section{Introducción}

El Marketing Estratégico se constituye en una herramienta gerencial que permite a las organizaciones educativas el desarrollo en el sector por medio del estudio del mercado, la identificación de necesidades para generar valor a los clientes, el aprovechamiento de las capacidades de la institución y la minimización de las debilidades. En este sentido, los cambios de la sociedad aumentan la competencia del mercado educativo, específicamente el de las Universidades Públicas, haciendo más difícil su posicionamiento. Por consiguiente, el propósito de la investigación que dio origen a este artículo era evidenciar la orientación del Marketing Estratégico en Universidades Públicas, reflejando cómo los paradigmas del entorno inducen a un mercado educativo moderno con oferentes que incrementan la competencia, haciendo más difícil el reconocimiento en el mercado universitario.

\section{Visión teórica}

\section{Marketing estratégico}

Para Sainz (2007:40), el marketing estratégico es un proceso responsable orientado a identificar, anticipar y satisfacer las necesidades del cliente, con la finalidad de fidelizarlo, de forma que la organización pueda obtener sus objetivos estratégicos. En este sentido, el rigor del mercado compuesto por clientes exigentes hace necesario la aplicación del marketing desde una perspectiva estratégica, donde se ofrezcan productos o servicios que guarden estrecha relación con el segmento y el contexto.

Por consiguiente, el mencionado autor plantea que el marketing ha evolucionado radicalmente para adaptarse al comportamiento del cliente. En los primeros tiempos de exceso de demanda, el proceso era pasivo, la empresa se centraba en el producto y en sus preocupaciones internas descuidando las expectativas de los clientes. Sin embargo, a medida que el cliente aumentaba las exigencias e información y se consolidaban los mercados maduros se ha hecho necesario dotar esta disciplina desde una dimensión estratégica.

En efecto, el marketing estratégico, dentro de su orientación, analiza la estrategia de segmentación como elemento clave, la intensidad de la competencia y la innovación tecnológica acelerada, entre otros aspectos, determinando la imposibilidad de ofrecer productos o servicios que no guarden relación con lo que el mercado necesita, sino que es imprescindible actuar en concordancia con lo que los consumidores esperan de la organización.

Al respecto, Munuera y Rodríguez (2007:54) proponen que el marketing estratégico viene caracterizado por el análisis y comprensión del mercado a fin de identificar las oportunidades que permiten a la empresa satisfacer las necesidades y deseos de los con- 
sumidores mejor y más eficientemente que la competencia. La importancia de este se ha incrementado progresivamente como consecuencia del análisis dinámico del atractivo del mercado, el proceso de segmentación, estudio de competidores y la naturaleza de la fuerza de la ventaja competitiva, entre otros.

Para los anteriores autores, la dirección del marketing estratégico comparte con la orientación global de la empresa el objetivo de lograr una ventaja competitiva, así como las funciones propias de toda dirección (sectores, líneas de productos y segmentos de mercado) y en la forma de perseguir este objetivo. En este sentido, el marketing estratégico se encarga de transmitir al conjunto de la organización el conocimiento del medio al que se enfrenta, es decir, el entendimiento sobre la evolución del mercado.

Por consiguiente, para desarrollar esta orientación al mercado, el marketing estratégico debe perseguir los siguientes objetivos: detectar nuevos segmentos o nichos con crecimiento potencial para desarrollar nuevos conceptos de producto, ampliar la oferta de productos según lo requiera el mercado, encontrar una ventaja competitiva sostenible y diseñar la estrategia de marketing adecuada.

Por otro lado, Cravens y Piercy (2007:624) se refieren al marketing estratégico como un proceso orientado al mercado donde se tiene en cuenta el entorno empresarial que cambia continuamente por la necesidad de ofrecer un valor superior para el consumidor. En este aspecto, Zeithmal (1998), citado por Soret (2008:37), argumenta que el valor percibido puede ser considerado como la valoración global que hace el consumidor de la utilidad con base en las percepciones de lo que recibe y lo que da. En este sentido, centra la atención en el rendimiento organizativo más que en las ventas, vinculando a la organización con el entorno, considerando el marketing responsabilidad de todo el negocio más que una función especializada.

De esta manera, el marketing estratégico orienta al mercado, establece estrategias y actividades de implementación para desarrollar una visión sobre los mercados de interés, segmentar, seleccionar estrategias al mercado objetivo, gestionar el posicionamiento y satisfacer los requisitos de valor de los consumidores en cada nicho de mercado. Es decir, el marketing estratégico proporciona el conocimiento necesario para hacer seguimiento del entorno, predecir a qué grupos de consumidores se va a atender, guiando las especificaciones de los productos y eligiendo ante qué competidores se va a posicionar.

\section{Perspectiva de la orientación al mercado}

La temática de la orientación al mercado (OM) tiene auge a partir de los años 90; las bases se fundamentan en los trabajos científicos que evidenciaban las acciones de las organizaciones guiadas por una filosofía de marketing y el aporte de esta en los resultados 
corporativos. Esto ha propiciado un interés por analizar dicho concepto desde diferentes perspectivas, pero a la vez coincidiendo en la necesidad de estudiar al cliente como el núcleo central de dicha filosofía; al respecto, se presenta la opinión de varios autores.

Según Kohli y Jaworski (1990), citado por Küster (2002:141), la orientación al mercado consiste en la generación de información del mercado que tenga que ver con las necesidades actuales y futuras de los clientes, la diseminación de toda ella a través de los distintos departamentos y la respuesta de la empresa a dicha información. En este sentido, los autores presentan algunos aspectos relevantes en el estudio del concepto sobre orientación al mercado, como son los antecedentes desde los factores de dirección, dinámica interdepartamental y los sistemas organizativos. Los mencionados autores concretan el grupo de factores relativos a la alta dirección desde la importancia que los directivos conceden a las necesidades de los consumidores; la dinámica interdepartamental, incluyendo componentes relativos al grado de conflicto y coordinación entre los departamentos, y el factor sistemas organizativos, con la existencia de ciertas características de la organización que potencialmente pueden interferir en la orientación al mercado.

Al respecto Narver y Slater (1990), citado por Roig, Ribeiro y otros (2004:315), argumentan que la orientación al mercado es la cultura organizativa que de manera más eficaz y eficiente determina los comportamientos necesarios en la creación de un valor superior para los consumidores. Así mismo, la información obtenida del consumidor, la competencia y los demás actores del entorno facilitan la generación de una respuesta proactiva.

De igual forma, cuando las empresas orientadas al mercado no solo analizan las fuerzas y debilidades a corto plazo, sino también las fortalezas y capacidades a largo plazo de la competencia, la cultura del aprendizaje deriva un comportamiento proactivo cuya característica primordial es que está dispuesta a desaprender todo lo que ya no es útil, e incorporar constructos necesarios para orientarse por el camino de los resultados de éxito, seleccionando los mercados a satisfacer y los grupos a controlar.

En este contexto, los clientes finales son quienes determinan el vencedor de las estrategias competitivas; la importancia para la actividad de la empresa se ha reconocido por décadas. El cliente intermediario o distribuidor constituye el primer cliente externo de la empresa; quienes permiten que los productos o servicios estén disponibles para los clientes finales, satisfacen la demanda a través de las actividades de promoción. La competencia está formada por los competidores que pueden interferir la satisfacción de los mercados constituyendo un moderador importante del rendimiento de la empresa y el macro entorno se evidencia como un fenómeno externo que influencia la efectividad organizativa. 
Así mismo, el uso organizacional de la información presenta diferentes perspectivas para medir su uso y coordinar las actividades competitivas de la empresa. Por tanto, si una empresa desea orientarse al mercado debe recoger información para las decisiones competitivas, constituyendo la primera etapa del marketing y el eje del marketing estratégico y la base de la ventaja competitiva sostenible, permitiendo reducir las variaciones macro ambientales. La coordinación interfuncional es la etapa que asegura la participación de las unidades organizativas en la creación de valor para los segmentos objetivos y en la rápida respuesta a sus demandas.

De igual forma, la coordinación es reconocida como requisito básico en una orientación al cliente y al mercado, pues provee información sobre las expectativas del mercado a las diferentes unidades organizativas, las acciones que la empresa dirige a sus mercados, competencia y macro entorno, que son el resultado de la coordinación basada en la información del mercado. Estas acciones buscan satisfacer tanto las necesidades del mercado como las de la organización y para ello estas acciones se distinguen por la anticipación y el cumplimiento de las expectativas generadas en los segmentos rentables.

Al respecto, Kotler y Armstrong (2008), citados por Esteban (2008:816), explican que la orientación al mercado facilita a las empresas la consecución de un doble objetivo: a) motivar a los empleados para servir bien a los clientes, y b) aumentando así la rentabilidad a través del establecimiento de relaciones sólidas de fidelidad con los mismos. Para ello es necesario contratar, entrenar y motivar al personal para que sirvan eficientemente a los clientes, es requisito indispensable para mantener relaciones satisfactorias con el entorno.

En este contexto, Hult y Ketchen (2000), citados por Munuera y Rodríguez (2007:41), proponen que el recurso empresarial fundamental para crear ventajas competitivas sostenibles en el tiempo es la orientación de mercado, siendo un factor determinante como elemento central desde la filosofía de la dirección, apoyada desde el concepto de marketing, el cual se puede afrontar desde la información, conocimiento e interpretación del mercado.

Por consiguiente, la orientación al mercado se operacionaliza en conductas o rutinas competitivas, obviando los aspectos ideológicos, concibiéndola como un constructo continuo, es decir, que el nivel de la orientación en las organizaciones varía en la magnitud en que ellas analizan el mercado, la competencia y el entorno, difundiendo información sobre la organización para coordinar la producción de la oferta y responder basados en la información provista por el diagnóstico y la coordinación organizacional.

Los anteriores autores fundamentan la orientación al mercado en dos posturas, desde la selección de los mercados para satisfacer la firma y el uso de la información en las organizaciones. De esta manera, la selección de los mercados y los grupos a controlar como 
lo son los clientes desde las necesidades, factores, cambios, nuevos segmentos, diferentes roles, posiciones de servicios, imagen, actitudes, oportunidades, amenazas y valor generado.

De igual forma, Metzger y Donaire (2007:179) explican que una orientación de mercado brinda la base para crea valor, tanto para los clientes como la empresa, al analizar el mercado en el que esta debe operar. Permitiendo a la organización la participación en mercados competitivos dinámicos mediante el reconocimiento de oportunidades en el mercado, así como las amenazas y la forma de equipararlas con las fortalezas de la organización, a la vez que minimiza el efecto de las debilidades.

En este sentido, la perspectiva basada en el concepto de mercadeo hace referencia a la cultura del marketing, basada en la orientación en el cliente, es decir, la orientación al mercado es una estrategia que la organización usa para obtener una ventaja competitiva sostenible, donde resulta del uso de recursos y habilidades para generar satisfacción diferencial en los mercados que son rentables. La ventaja se convierte en sostenible porque las conductas recurrentes asociadas con la orientación al mercado requieren de un complejo aprendizaje organizativo que no puede ser imitado fácilmente por los competidores.

Por consiguiente, según los autores citados la orientación al mercado determina el ajuste apropiado de la empresa con el ambiente de negocios mediante el análisis detallado de la situación, establece el valor a ofrecer a clientes específicos mediante la selección de mercados objetivos apropiados, ofrece el producto o servicio, la fijación de precios, los canales apropiados y la comunicación a través de promociones de la empresa. Así mismo, es considerada por algunos como una cultura organizativa fundamentada en la motivación de los empleados hacia la prestación de un excelente servicio a los clientes. Sin embargo, se encuentran diferencias en cuanto al aporte desde el análisis del mercado con el fin de obtener información de este, permitiendo a la empresa entender y satisfacer las necesidades de los consumidores y establecer ventajas competitivas sólidas en el contexto donde actúa.

\section{Perspectiva cultural}

Bajo la óptica de Munuera y Rodríguez (2007:42), el enfoque de la perspectiva cultural dimensiona la orientación al mercado como una cultura organizativa que genera las actitudes necesarias para la creación de un valor superior para los consumidores, maximizando las oportunidades de aprendizaje del mercado y la disposición a compartir la información, identificando las normas y valores de la organización.

Desde la perspectiva de Hurley y Hut (1998), citados por Martínez (2004:145) argumentan que la perspectiva cultural puede concretarse diciendo que es la manifestación más profunda de la orientación al mercado pues es desde la cultura, donde con el paso 
del tiempo los acontecimientos, el reforzamiento del comportamiento y la creación de procesos organizativos, crean el convencimiento fundamental en los empleados de que los clientes y el aprendizaje sobre el mercado son importantes. Es decir, este concepto representa un conjunto de valores y actitudes compartidos en toda la organización, estimulando la creación de valor para los clientes.

Al respecto Narver y Slater (1990), citado por Munuera y Rodríguez (2007:42), fundamentan la perspectiva cultural desde tres componentes: la orientación al consumidor, la orientación al competidor y la coordinación interfuncional. Por lo tanto, si una orientación al mercado fuera simplemente un conjunto de actividades completamente desasociadas del sistema subyacente de creencias de una organización, entonces, cualquiera que fuese la cultura de la organización, una orientación al mercado podría ser implantada en cualquier momento por la organización, pero eso no es lo que se observa.

Partiendo de los enunciados anteriores, los autores coinciden en el hecho de que la perspectiva cultural enmarca el conjunto de valores, costumbres, actitudes y aprendizaje compartidos por la organización para el entendimiento del mercado, con el fin de estimular la creación de valor en los clientes. En este sentido, se puede inferir que la perspectiva cultural es el mecanismo integrador de creencias y subjetividad de los individuos como parte del estudio del conjunto de actividades organizacionales.

De manera tal, el estudio de la perspectiva cultural juega un papel relevante en el desarrollo empresarial desde la orientación al mercado, pues son las personas los agentes activos de la actividad empresarial, quienes en la cotidianidad aportan al desarrollo organizacional un conjunto de factores distintivos de cada micro entorno, como lo son las creencias, valores, hábitos y costumbres que de una u otra forman repercuten en la consecución de los objetivos organizacionales.

\section{Perspectiva comportamental}

Kohli y Jaworski (1990), citados por Munuera y Rodríguez (2007:54) argumentan que el enfoque de la perspectiva comportamental de la orientación al mercado es asimilable al procesamiento de la información al mercado. En este sentido los mencionados autores conceptúan la orientación al mercado en torno a tres elementos: la generación de inteligencia de mercado, diseminación por el conjunto de departamentos y la respuesta de toda la organización.

Por otro lado, Matsuno y Mentzer (2000), citados por Martínez (2004:145), consideran que la perspectiva comportamental tiene como base fundamental las actividades o proceso de información del mercado o inteligencia del mercado, dimensionándose con 
la generación, diseminación y respuesta de esta inteligencia, pero además ańaden los factores de influencia macro ambientales, las tendencias socioculturales de los segmentos del mercado, estilo de vida, regulación del ambiente y aspectos laborales, entre otros.

Al respecto Fernández Mavondo y Farell (2000), citados por Martínez (2004:146), argumentan que la perspectiva comportamental juega un papel importante en la orientación del mercado al establecer las normas de generación y respuesta de información del contexto pero a su vez le atańen un nuevo concepto desde el entendimiento del mercado.

De acuerdo con el planteamiento de los autores, la perspectiva comportamental de la orientación al mercado es correspondiente con el procesamiento de la información al mercado, teniendo en cuenta para el óptimo entendimiento: la generación de inteligencia del mercado, diseminación por el conjunto de departamentos y la respuesta de toda la organización, remitiendo a las acciones organizacionales el cúmulo de información suministrada sobre el mercado.

\section{Proceso fenomenológico sobre la orientación del marketing estratégico en universidades públicas}

El proceso de la investigación cualitativa es planteada por Rodríguez y otros (1996), citados por Martínez (2007:351), como el conjunto de observadores competentes y cualificados que informan con objetividad, claridad y precisión acerca de las propias observaciones del investigador sobre el mundo social, así como de las experiencias de los demás, aproximándose a un sujeto real, que está presente en el mundo y que puede ofrecer información sobre sus propias experiencias, opiniones, por medio de un conjunto de técnicas o métodos, como las entrevistas, historias de vida, estudios de casos, análisis documental, entre otros.

En este contexto, se infiere que los investigadores cualitativos escogen el método que les permita registrar sus propias observaciones de una forma adecuada, colocando al descubierto los significados que los sujetos ofrecen de sus propias experiencias, confiando en las expresiones subjetivas, escritas y verbales, de los significados dados por los sujetos estudiados, adentrándose en el interior de cada situación o sujeto. Sin embargo, Rodríguez y otros (1996), citados por Martínez (2007:351), manifiestan que los investigadores cuando realizan una investigación cualitativa, no siempre operan siguiendo un esquema de acción determinado, y cuando tal esquema existe tampoco es el mismo para ellos.

A continuación, se presenta una propuesta de lo que se considera proceso de investigación cualitativa, aplicada en la investigación que dio origen al presente artículo. Se expresa a través del proceso de investigación cualitativa (ver gráfico 1) con una serie de 
fases y etapas que se superponen unas con otras, orientadas a responder los propósitos del estudio de la Orientación del Marketing Estratégico en Universidades Públicas. Para el cual se establecieron cuatro fases fundamentales:

- Fase I: Preliminar

- Fase II: Acceso al escenario de investigación

- Fase III: Analítica

- Fase IV: Difusión

Gráfico 1. Fases del proceso de investigación cualitativa.

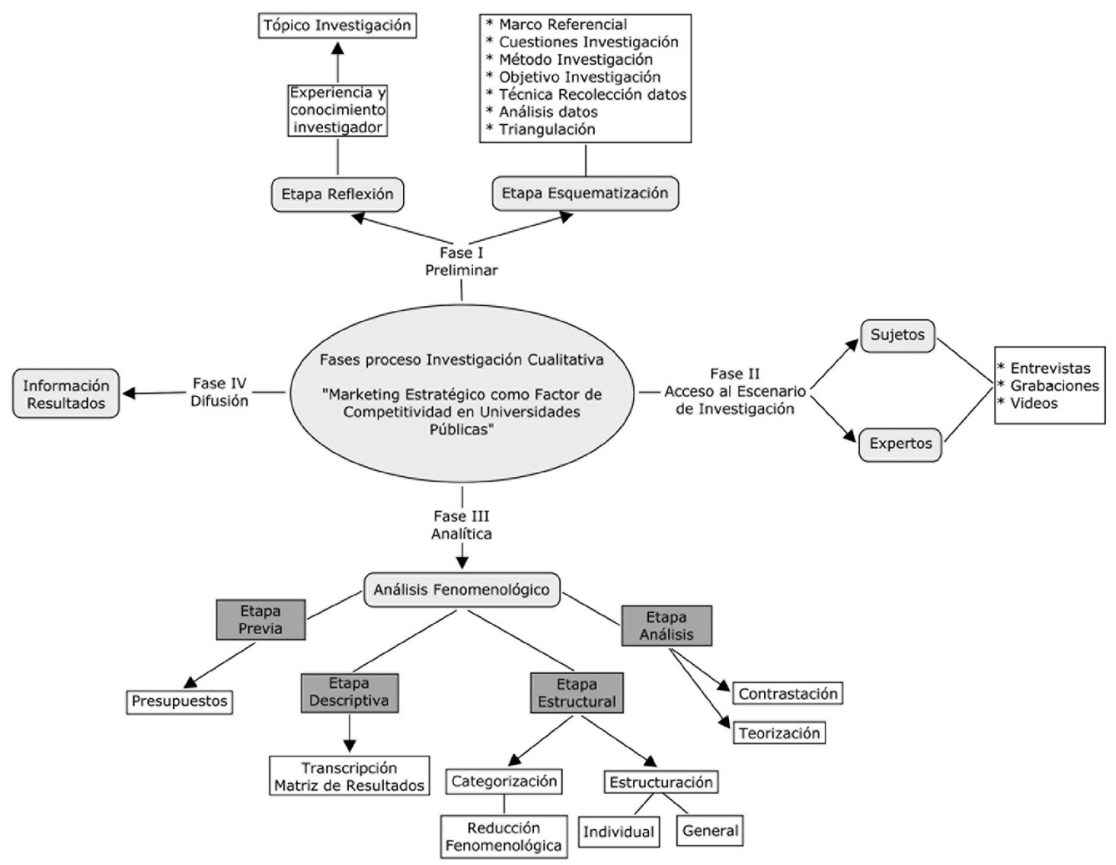

Fuente: elaboración propia.

\section{- Fase I Preliminar}

En esta primera fase se establecen dos etapas. En la primera etapa denominada reflexión, se tomó en cuenta la experiencia y conocimiento de la investigadora, donde se trató de clarificar y determinar el tópico de interés describiendo las razones por las que elige el tema. Posteriormente, identificado el tópico, se busca toda la información posible sobre el mismo, se trata de establecer un marco referencial sin llegar a extremos. Des- 
pués sigue la etapa de esquematización que consiste en planificar las acciones, es decir, diseñar la investigación.

\section{- Fase II Acceso al escenario de investigación}

En este momento, la investigadora tiene acceso al escenario de investigación de una forma progresiva, estableciendo contacto con los sujetos y expertos de la Universidad Nacional Experimental del Táchira y Universidad de Pamplona. Para recoger y registrar la información se utilizaron diferentes sistemas como videos y grabaciones de audio. Inicialmente, la información fue amplia, recopilando todo; progresivamente se fue focalizando hacia una información mucho más específica, desarrollándose en un contexto de interacción personal. De esta forma, la investigadora define el rol según el grado de participación (investigador) y así mismo los sujetos del escenario de estudio van definiendo su papel según el grado de información suministrada (sujetos o expertos).

\section{- Fase III Analítica}

El análisis de datos cualitativos se considera como un proceso con cierto grado de sistematización, que en oportunidades permanece implícito en las actuaciones de la investigadora. En este aspecto, es difícil definir una estrategia para el análisis de los datos cualitativos; sin embargo, para la presente investigación se definen las siguientes etapas correspondientes al análisis fenomenológico: etapa previa (clarificación de los presupuestos de los cuales parte el estudio, etapa descriptiva (transcripción fidedigna de lo expresado por entrevistados en matriz de resultados), etapa estructural compuesta por la categorización (desarrollo de análisis de contenido; reducciones fenomenológicas) y la estructuración (individual y general) y la etapa de análisis compuesta por la contrastación (triangulación: sujetos, expertos, teoría) y la teorización (resultado de la teoría emergente).

\section{- Fase IV Difusión}

El proceso de investigación culmina con la presentación, difusión e información de los resultados. En esta fase se alcanza una mayor comprensión del fenómeno objeto de estudio por parte de la investigadora, quien comparte la información con los demás.

\section{Estructura cognitiva integradora del marketing estratégico en universidades públicas}

A continuación se presenta el producto del conocimiento científico planteado en este artículo como resultado de las reflexiones e interpretaciones de las entrevistas y videos 
aplicados en la investigación, sustentado en el análisis de las relaciones de la realidad específica del marketing estratégico en universidades públicas, delineando las conclusiones generales de la temática desarrollada.

Gráfico 2. Estructura cognitiva integradora.

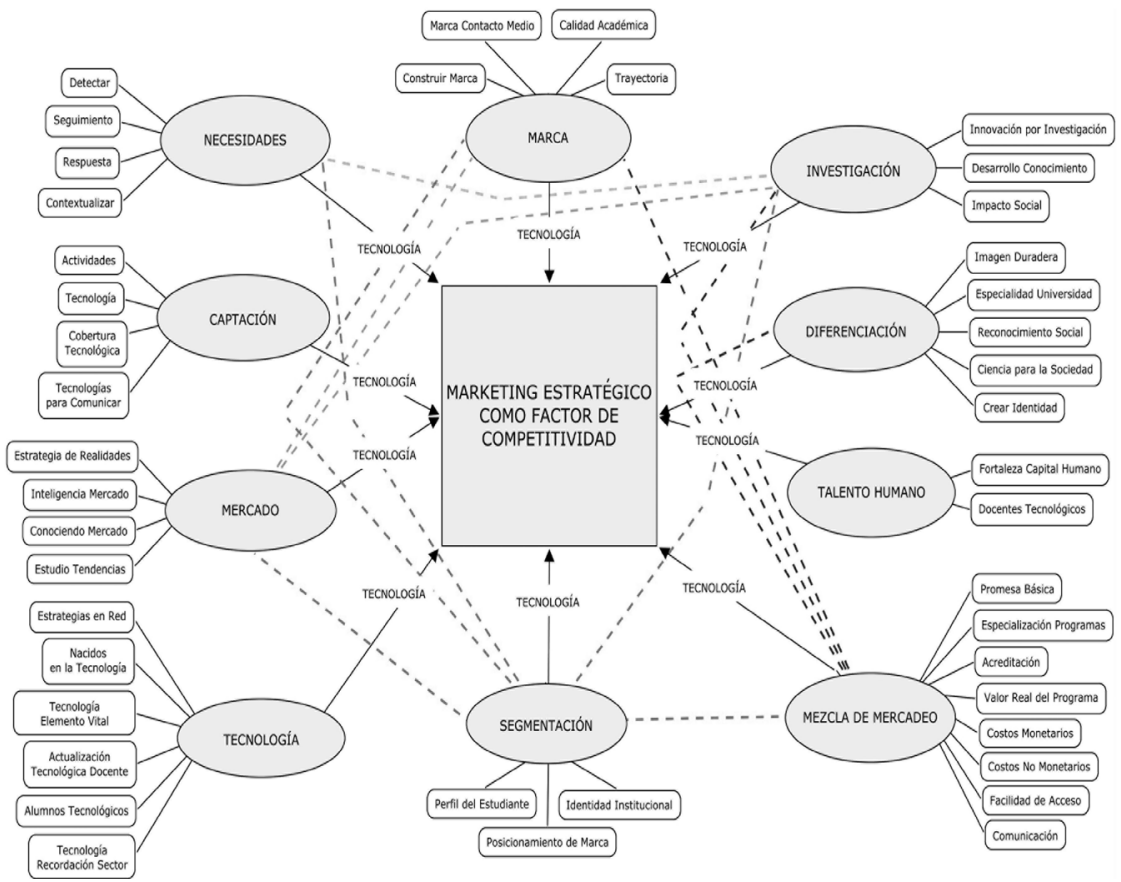

Fuente: elaboración propia.

Tomando como base el gráfico 2 cognitivo integrador, se afirma que la actitud de los sujetos y expertos con respecto al marketing estratégico en universidades públicas, se evidencia en la reflexión de aspectos como las necesidades, captación, mercado, tecnología, segmentación, mezcla de mercadeo, talento humano, diferenciación, investigación y marca. Así mismo, los hallazgos de la investigación que dio origen a este artículo permiten establecer que la actitud de las instituciones hacia el marketing estratégico está influenciada por criterios conservadores que imposibilitan su desarrollo. Por lo anterior, las instituciones deben acondicionar su funcionamiento a partir de los siguientes componentes:

- Conocer al estudiante

- Conocer al mercado

- Conocer las tendencias tecnológicas

- Conocer las capacidades institucionales 
Teniendo en cuenta lo planteado por Sainz (2007:40), el marketing estratégico es un proceso responsable orientado a identificar, anticipar y satisfacer las necesidades del cliente, con la finalidad de fidelizarlo, de forma que la organización pueda obtener sus objetivos estratégicos; en este sentido, el rigor del mercado educativo compuesto por clientes exigentes hace necesario en las universidades públicas la aplicación del marketing desde una perspectiva estratégica donde se ofrezcan productos o servicios que guarden estrecha relación con el segmento y el contexto, contribuyendo a la solución de las problemáticas sociales, el desarrollo de la empresa y la potencialización de la investigación.

\section{Factores claves de éxito de las universidades públicas}

La investigación llevada a efecto por el autor, evidencia que las universidades públicas deben considerar factores claves de éxito para el desarrollo del marketing estratégico como factor de competitividad. Dentro de los cuales se destacan:

Certificación de calidad: Certificación de máxima calidad, denominada acreditación.

Pertinencia con el medio: Compromiso con el medio desarrollado a través de la docencia, la investigación y la extensión.

Alianzas empresariales: Establecimiento de convenios de cooperación en materia de investigación y desarrollo.

Infraestructura: Adecuación de la capacidad instalada de la Universidad para cumplir con sus principios misionales.

Investigación: Reconocimiento por el impacto social derivado de la investigación.

Internacionalización: Creación de convenios de cooperación en temas académicos e investigativos.

Imagen de la universidad: Consolidación de la imagen en el medio.

Tecnología: Transformación tecnológica de las instituciones universitarias.

Posicionamiento de marca: Establecimiento de ventajas competitivas que identifiquen la institución.

Conocimiento del estudiante: Detección, seguimiento, respuesta y contextualización de las necesidades de los estudiantes.

Portafolio de programas: Desarrollo de programas certificados por normas de calidad que garanticen la pertinencia con el medio. 


\section{Puntos débiles de las universidades públicas}

Las universidades públicas deben contrarrestar los puntos débiles para el desarrollo del marketing estratégico, dentro de los cuales se destacan:

- Escasez de recursos disponibles para promocionar los servicios de la institución.

- Ausencia de Acreditación y vencimiento de registros de calidad de programas académicos.

- Falta de estrategias para atender la diversidad de población estudiantil.

- Carencia de estrategias que contribuyan a disminuir la deserción de los estudiantes.

- Poca preparación tecnológica de los docentes.

- Falta conexión de la imagen de la universidad con el entorno.

- Desconocimiento de la competencia.

- Carente desarrollo de transferencias tecnológicas (conocimiento).

- Ausencia de Estrategias de Impacto al contexto social.

- Carencia de estudio de tendencias del mercado.

- Falta de diseńo del plan estratégico del mercadeo.

\section{Consideraciones finales}

El desarrollo de la sociedad conduce a las Universidades Públicas a enfrentarse a un mercadeo educativo caracterizado por el aumento de la competencia, reduciendo la cantidad de alumnos, dificultando el posicionamiento en el sector. En este sentido, las instituciones de educación superior han incorporado incipientemente el marketing, limitándose a la aplicación de publicidad, desconociéndose el poder que el mismo tiene en la determinación de necesidades de la organización y el crecimiento. En el proceso de la presente investigación se evidenció que las Universidades Públicas orientan parte de sus acciones a la vivencia del marketing estratégico, constituyéndose en el factor de competitividad, lo cual se pudo corroborar en las entrevistas realizadas a los sujetos expertos y videos de las dos universidades estudiadas.

Así mismo, las entrevistas y videos revelaron que estas instituciones públicas contemplan aspectos como la perspectiva cultural y la perspectiva comportamental, destacándose las actividades de atención al estudiante, compromiso de las dependencias, captación, seguimiento y contacto permanente; sin embargo es imperativo realizar un proceso 
de detección, seguimiento, respuesta y contextualización de las necesidades con el fin de direccionar el segmento de la universidad y definir una identidad institucional proyectando a las instituciones socialmente mediante la utilización de la tecnología.

Por consiguiente, la segmentación trabajada desde el establecimiento de criterios de selección, clasificación, determinación de características, segmentos y preferencias, estableciendo grupos objetivos por atender. En este sentido, las universidades públicas deben delinear su propio perfil de estudiante conectándolo con la realidad del medio donde se desarrolla, por consiguiente con la identidad institucional, alcanzando la consolidación de la marca. De igual forma, en la mezcla de mercadeo que se presenta en estas instituciones se vislumbran elementos como el producto, precio, plaza y promoción; dicha mezcla se evidencia desde el portafolio de programas, la promesa básica, el valor real del programa, la cobertura y los medios de comunicación entre otros aspectos; sin embargo, es importante que el adecuado desarrollo de la mezcla de mercadeo contribuya a la consolidación de la especialización institucional a partir del estudio de tendencias y la incorporación de la tecnología.

En este contexto, las universidades públicas deben generar un ambiente de búsqueda, análisis, interpretación y divulgación de la información acerca de los diferentes mercados con el fin de que la institución aproveche las fortalezas, mejore las debilidades, determine las oportunidades, y contrarreste las amenazas de un entorno actual altamente competitivo, buscando como fin máximo la satisfacción de las necesidades de los estudiantes de la forma más eficaz y eficiente posible.

\section{Bibliografía}

Cravens, David y Piercy, Nigel (2007). Marketing estratégico. Madrid - España, McGraw Hill. $8^{a}$ edición, p. 624.

Esteban, Águeda y otros (2008). Principios de marketing. Madrid - España, ESIC. Libros profesionales de empresa. 3a edición, p. 816.

Kuster, Inés (2002). La venta relacional. Madrid - España, ESIC. Escuela Superior de Gestión Comercial y de Marketing p. 141.

Martínez, María (2004). Orientación a Mercado. Un modelo desde la perspectiva de aprendizaje organizacional. México, Universidad Autónoma de Aguas Calientes. $1^{\mathrm{a}}$ edición, p. 145, 146.

Martínez, Migueles (2007). Ciencia y arte en la metodología cualitativa. México, Editorial Trillas, p. 351.

Metzger, Michael y Donaire, Víctor (2007). Gerencia estratégica de mercadeo. México, Thomson Editores, p.179. 
Munuera, José y Rodríguez, Ana (2007). Estrategias de Marketing: Un enfoque basado en el proceso de dirección. Madrid - España, ESIC. Alfaomega, p. 41, 42,54.

Roig, Ribeiro y otros (2004). El emprendedor innovador y la creación de empresas de I+D+I. España, Edición Universidad de Valencia, p. 315.

Sainz, José (2007). El plan de marketing en la práctica. Madrid - España, ESIC. Libros profesionales de empresa. $11^{\text {a }}$ edición, p. 40.

Soret, Ignacio (2008). Modelo de medición de conocimiento y generación de ventajas competitivas. Madrid - España, ESIC. Alfaomega, p. 37.

\section{Cómo citar este artículo:}

Santafé, Akever (2014). "Orientación del marketing estratégico en universidades públicas”, Oikos No 37, 25-39, Escuela de Administración y Economía, Universidad Católica Silva Henríquez (UCSH), Santiago de Chile. [http://ediciones.ucsh.cl/revistas.php]

Fecha de recepción: 06 / 04 /2014

Fecha de aceptación: 14 / 11 / 2014 